\title{
C-reactive protein - biological functions, cardiovascular disease and physical exercise
}

\author{
S J Semple (DTech) \\ Department of Sport and Physical Rehabilitation Sciences, Tshwane University of Technology, Pretoria
}

\begin{abstract}
C-reactive protein $(\mathrm{CRP})$ is an acute-phase reactant that increases in response to noxious stimuli that inevitably induce cellular and/or tissue injury. The increased synthesis of CRP occurs predominantly in the liver and peaks 24 48 hours after the inciting stimulus. CRP forms an integral component of innate immunity and serves primarily to recognise potential pathogens and damaged cells. It facilitates the removal of these cells through opsonisation and activates the complement system. With increasing evidence supporting the classification of artherosclerosis as inflammatory in nature, CRP has received considerable attention as a marker, and in some cases a contributor towards this cardiovascular disease. Traditionally, CRP has been measured within exercise studies to provide evidence that an acute-phase inflammatory response can or has been initiated. Although the elevation in CRP following exercise has largely been attributed to muscle damage, evidence is mounting to contest this premise. Participation in chronic exercise has been associated with a reduced risk of cardiovascular disease. Numerous studies have now shown an inverse relationship between physical activity levels and resting concentrations of CRP. Thus, exercise may prove beneficial in lowering systemic inflammatory markers such as CRP, and consequently contribute towards preventing the progression of inflammatory disorders.
\end{abstract}

\section{Introduction}

C-reactive protein (CRP) was first discovered in 1930 by William Tillet and Thomas Francis. ${ }^{48}$ In studying the blood of patients suffering from acute Streptococcus pneumonia infection, it was found that the sera of these patients formed

\section{CORRESPONDENCE:}

\section{S J Semple}

Department of Sport and Physical Rehabilitation Sciences

Tshwane University of Technology

Private Bag X680

Pretoria

0001

Tel: 012-318 4324

Fax: 012-318 5801

E-mail: semplesj@tut.ac.za a precipitin with an extract from the streptococcal bacterium. The extract was originally labelled Fraction $\mathrm{C}$, and was later confirmed as a polysaccharide. Hence, as a result of its reactivity with the C-polysaccharide of the Streptococcus cell wall, the 'substance' in the sera was named CRP.

CRP ligand-binding is calcium dependent and binds with highest affinity to phosphocholine (PC), a constituent of the cell membrane phospholipid, phosphatidylcholine. Under normal conditions phophatidylcholine is not exposed, however, once a cell has been damaged it becomes 'accessible' to CRP.

\section{Synthesis and application}

CRP synthesis was originally thought to be confined to the liver with no evidence supporting its production in cells other than hepatocytes. ${ }^{35}$ Jabs et al. ${ }^{15}$ have recently shown by real-time polymerase chain reaction $(\mathrm{PCr})$ and immunohistochemistry, that in response to stimulation with interleukin6 (IL-6), renal cortical tubular epithelial cells express CRP messenger ribonucleic acid (mRNA). Additional extrahepatic sites of CRP synthesis/gene expression have been identified and include the epithelial cells of the human respiratory tract and T-lymphocytes in culture. ${ }^{13,14}$ It is still generally accepted that the liver is the primary site of de novo CRP production.

CRP concentration can increase dramatically up to $1000-$ fold during the acute-phase response, and usually peaks 24 - 48 hours after an initial acute inflammatory stimulus. ${ }^{20,34}$ The half-life of CRP is 19 hours and is independent of the circulating concentration of CRP. ${ }^{1}$ Hence, the primary factor determining the serum level of CRP is the rate at which it is produced. ${ }^{1}$

Normal systemic CRP levels are classified as less than $5 \mathrm{mg} / \mathrm{l}$ with averages of the sedentary/general population being estimated at approximately $2 \mathrm{mg} / \mathrm{l}^{9}$ No difference in concentration exists between males and females, nor does CRP exhibit diurnal or seasonal variation. ${ }^{6,34}$ Serum levels are not affected by food intake, however, Ganapathi et al. ${ }^{10}$ have shown that the CRP levels in human hepatoma cell lines are potentiated by caffeine. In addition, Church et al. ${ }^{5}$ have shown that CRP levels are reduced through the use of a multivitamin over a 6-month period.

Regardless of the nature and location of cellular/tissue damage, a non-specific, systemic acute-phase response is initiated. Although CRP has been identified as one of the most prominent acute-phase proteins that allows for quantification 
of an inflammatory state, its elevation cannot differentiate between damaged tissues, hence its measurement and diagnostic value has been questioned. ${ }^{1}$ Thus, CRP is generally measured within a clinical setting to provide the physician with an indication of disease activity, the effectiveness of pharmacological treatment and to determine if intercurrent infections have manifested. ${ }^{1}$ Whilst an increase in CRP has generally been accepted as a response to an inflammatory 'condition', Kushner has proposed that minor elevations of CRP are indicative of biological ageing, a non-inflammatory condition. $^{19}$

\section{Biological properties and functions of CRP}

\section{Recognition of pathogens and damaged cells}

As part of the acute-phase response, CRP levels may rise dramatically in order to facilitate non-specific immune functions and assist with the repair process. The ability of CRP to recognise disease-causing agents and damaged cells, and to mediate their removal, in conjunction with the fact that there exists an absence of any documented human CRP deficiency, highlights its crucial role in innate immunity.

Within the human body millions of cells die each day. Gershov et al. ${ }^{11}$ have proposed that a key role of CRP is to facilitate the removal of these cells. They reported that in addition to binding to lysed or permeabilised cells, CRP binds to the membranes of intact apoptotic cells. The increased CRP was associated with enhanced phagocytosis of the apoptotic cells and would thus contribute towards their clearance.

\section{Opsonisation}

On binding to various cell membrane surfaces or necrotic tissue/debris, CRP then acts as an opsonin (from the Greek meaning 'prepare food for'). ${ }^{2}$ Opsonisation involves coating of the bacterial surface so that it can be recognised by other cells of the immune system, specifically macrophages and neutrophils. Thus, opsonisation by CRP promotes the uptake, and therefore removal of these cells by phagocytes.

\section{Activation and regulation of complement pathways (pro v. anti-inflammatory role)}

In addition to the binding and subsequent opsonisation of pathogens, CRP also serves to activate and modulate complement. CRP binds to $\mathrm{C} 1 \mathrm{q}$, the first component of the complement cascade, and thereby initiates activation of the classical pathway. The activation of complement serves to enhance opsonisation and increase local inflammation. Berman et al. ${ }^{3}$ have shown that although CRP activates the classical pathway of complement, the terminal components (C5C9) known as the membrane attack complex (MAC) are not activated. This was in contrast to immunoglobulin $G$ ( $(\mathrm{gG})$ and immunoglobulin $\mathrm{M}$ (IgM) activation of the classical pathway, which formed the MAC. The non-activation of the MAC could be interpreted as an anti-inflammatory mechanism, since activation of these terminal components has been as- sociated with cellular injury and the release of pro-inflammatory cytokines.

CRP also inhibits the alternate and lectin pathways of complement through the recruitment of factor $\mathrm{H}$, a regulatory protein that promotes the degradation of the C3 and C5 convertase. ${ }^{27}$ Thus CRP plays a dual pro and anti-inflammatory role in its regulation of the complement system.

The immunomodulating actions of CRP and its pro and anti-inflammatory effects are not restricted to complement. $\mathrm{Pue}^{37}$ has shown that in response to lipopolysaccharide (LPS), the hosts' peripheral blood mononuclear cells (PBMC) respond to $\mathrm{CRP}$ in a pro-inflammatory manner. However, once the CRP has moved into the tissue and reacts with macrophages, inflammation is suppressed through the inhibition of interleukin-1b (IL-1b) and interleukin-1ra (IL1ra). Additional anti-inflammatory properties exhibited by CRP include the ability to decrease the expression of cell adhesion molecule (L-selectin) in vitro, and reduce neutrophil superoxide production. ${ }^{9,54}$

\section{CRP and cardiovascular disease}

A plethora of studies have recently associated elevated serum CRP levels with an increased risk of developing cardiovascular disease (CVD). The increase in CRP is indicative of an inflammatory response, and it is now widely accepted that atherosclerosis (the underlying cause of most CVD) is a chronic inflammatory disorder. ${ }^{38}$

Atherosclerosis is in part characterised by the deposition and accumulation of lipids within arterial walls. These lesions can lead to ischaemia of the brain, heart and peripheral tissues resulting in infarction. ${ }^{38}$ The oxidation of low-density lipoprotein (LDL) deposited on arterial walls is one of a number of factors contributing to what has been termed endothelial dysfunction, ${ }^{38}$ a hypothesis proposing that vascular injury, and hence inflammation, is induced by a number of factors and possibly a combination thereof. Possible causes of endothelial dysfunction are elevated and modified LDL, diabetes, genetic alterations, cigarette smoking, hypertension, elevated homocysteine, and infectious microorganisms (e.g. Chlamydia pneumoniae). ${ }^{38}$

Although substantial evidence from numerous studies has identified CRP as a marker of CVD, increasing evidence is now implicating CRP as a risk factor directly involved in atherogenesis. Cermak et al. ${ }^{4}$ have reported that CRP induced a 75-fold increase in tissue factor (TF) procoagulant activity of PBMC. It was suggested by the authors that the increase in monocyte TF expression (during infection/ necrosis) induced by CRP could contribute towards the development of intravascular coagulation. This could arguably exacerbate the inflammatory state already present in 'injured' vessels. Nakagomi et al. ${ }^{29}$ also reported increases in PBMC TF concentrations in response to stimulation with CRP. The authors proposed that their findings shed light on the link between inflammation and coagulation, a connection which may contribute to the progression and outcome of 
thrombotic events associated with atherosclerosis'.

Using human umbilical vein endothelial cells, Pasceri et al. ${ }^{31}$ showed that CRP induced the expression of monocyte chemoattractant protein-1 (MCP-1), a chemokine that attracts monocytes, natural killer (NK) cells and activates macrophages. The same group reported a CRP-induced increase in the expression of intercellular adhesion molecule1 (ICAM-1) and vascular cell adhesion molecule-1 (VCAM-1) in umbilical vein as well as coronary artery endothelial cells. ${ }^{32}$ Inhibiting the expression of cellular adhesion molecules has been shown to decrease phagocytic activity within atherosclerotic plaque. ${ }^{33}$ Pasceri et al. ${ }^{32}$ concluded that CRP is not just a marker of inflammation, but rather that it may contribute to enhancing the progression of inflammation/ atherosclerosis.

In keeping with the role that CRP plays in contributing towards atherogenesis, Zwaka et al. ${ }^{55}$ demonstrated that CRP facilitates the phagocytosis of native LDL by macrophages. The uptake of LDL particles by macrophages results in the formation of lipid peroxides, promotes the accumulation of cholesterol esters and eventually forms foam cells. ${ }^{38}$ Zwaka et al. $^{55}$ suggested that the binding of CRP to LDL within arterial walls might promote the onset of arteriosclerosis.

Nitric oxide (NO) plays an integral role in inflammation and immune regulation with lowered levels associated with adverse cardiac events. Verma et al. ${ }^{51}$ incubated endothelial cells with a concentration of recombinant CRP known to predict adverse cardiovascular events. They measured the production of $\mathrm{NO}$ and cyclic guanosine monophosphate (cGMP), the second messenger for NO. CRP elicited a dose-dependent, significant decrease in both $\mathrm{NO}$ and cGMP production. The authors concluded that the inhibitory effect that CRP exerts on NO production may facilitate the development of cardiovascular disease. Similarly, Venugopal et al. ${ }^{50}$ investigated the effects that CRP had on endothelial nitric oxide synthase (eNOS) expression. Human aortic endothelial cells (HAEC) were incubated with recombinant CRP, and in a finding mirroring that of Venugopal et al., ${ }^{50}$ eNOS and eNOS mRNA protein levels were inhibited by CRP. This finding further supports the role of CRP in the artherogenic process.

Yasojima et al. ${ }^{53}$ used a reverse transcriptase-polymerase chain reaction to detect $\mathrm{mRNA}$ for $\mathrm{CRP}$ and complement proteins (C1-C9) in arterial and atherosclerotic plaque. They found evidence of CRP as well as C1-C9 being synthesised within the arterial tissue of 10 postmortem cases. In addition, mRNA and the respective proteins were elevated in atherosclerotic plaque. Since complement proteins have been associated with atherosclerotic plaque, ${ }^{39}$ and CRP is instrumental in potentiating local inflammation by binding with $\mathrm{C} 1 \mathrm{q}$ it seems tenable that CRP may be involved in the pathogenesis of atherosclerosis.

\section{Inflammation and exercise}

It has been proposed that physical activity may serve as a model for studying the inflammatory response, ${ }^{41}$ and it is well established that an acute bout of exercise may alter the circulating levels of a number of pro-inflammatory cells including cytokines, acute-phase proteins and white blood cells. ${ }^{25,40,45}$ Although there is general consensus regarding the induction of an acute-phase inflammatory response following strenuous, unaccustomed or prolonged bouts of exercise, there is some degree of uncertainty surrounding the precise stimuli responsible for this response. The majority of authors have attributed the rise in inflammatory markers following physical activity to muscle damage. Evidence suggesting a possible role of other factors/stimuli involved in inducing or exacerbating the inflammatory response following exercise may include haemolysis, ${ }^{21}$ endotoxaemia, ${ }^{16}$ and the production of reactive oxygen species. ${ }^{17}$ In addition, psychological stress, which may be more prevalent in elite athletes, has also been shown to cause an increase in pro-inflammatory cytokines. ${ }^{22}$ Invariably, the mode, duration and intensity of the exercise, as well as the subject's level of conditioning may all affect the magnitude of the inflammatory response as well as resting concentrations of inflammatory markers such as CRP. In keeping with this, King et al. ${ }^{18}$ have proposed that certain activities such as jogging, may be more beneficial in terms of lowering inflammatory markers than other modes of exercise.

As outlined above, CRP is involved in the activation of the classical pathway of complement. Complement proteins are intimately involved in opsonisation, inflammation and cell lysis, ${ }^{28,42}$ and have been investigated in response to exercise of varying mode and duration. Similar to CRP, the specific stimuli that upregulate complement proteins following exercise are controversial. However, as with CRP the resting concentrations of selected complement proteins have been shown to be lower in athletes compared with sedentary individuals. ${ }^{30}$ More specifically, Nieman et al. ${ }^{30}$ reported lower levels of C3 in athletes compared with sedentary controls. Interestingly, elevated C3 has recently been proposed as a marker to identify the progression of atherosclerosis. ${ }^{46}$

\section{Effects of acute exercise on CRP}

The acute response of CRP to physical activity has been published extensively, and the following section outlines a few of the common findings. Increases in CRP have been observed following acute strenuous, prolonged bouts of running, ${ }^{40}$ triathlon, ${ }^{16,47}$ bench stepping ${ }^{12}$ and anaerobic exercise. $^{26}$ Since eccentrically based exercise is more commonly associated with muscle damage, and CRP serves to bind damaged cells, it seems reasonable to assume that this type of activity would be associated with pronounced elevations in CRP. Results contradicting this assumption have been documented by Sorichter et al. ${ }^{44}$ and Malm et al. ${ }^{23}$ In both studies no significant elevations were observed for CRP following eccentric exercise (70 eccentric quadriceps contractions, and 45 minutes of downhill running respectively). These results suggest that elevations in CRP following exercise may not be solely due to muscle damage. Supporting this would be the findings of Smith et al. ${ }^{43}$ In this study, CRP was significantly 
$(p<0.04)$ elevated in $75 \%$ of active-untrained subjects, 24 hours after performing 60 minutes of cycling at only $60 \%$ of maximal oxygen uptake. Similarly, Meyer et al. ${ }^{26}$ reported a significant $(p=0.02)$ increase in CRP 24 hours after 12 trained males performed an anaerobic cycle ergometer test.

\section{Effects of chronic exercise on CRP}

Pitsavos et al. ${ }^{36}$ used a sample of 891 men and 965 women older than 18 years, to determine the association of leisuretime physical activity on CRP and other inflammatory markers. The results revealed that CRP levels were 33\% lower in the subjects who partook in high-physical activity levels compared with the sedentary group (high-physical activity was defined as expended calories $>7 \mathrm{kcal} / \mathrm{min}$ ). Similarly, our laboratory has observed (unpublished data) that CRP resting levels are significantly lower in professional cyclists compared with active-untrained individuals.

Tomaszewski et al. ${ }^{49}$ have reported 'strikingly' low CRP levels in runners. Sixty-seven male ultra-marathon runners were compared with sedentary individuals. They were all categorised into groups having a body mass index (BMI) less than $25 \mathrm{~kg} / \mathrm{m}^{2}$ or greater than $25 \mathrm{~kg} / \mathrm{m}^{2}$. Although nonsignificant, the resting levels of CRP were markedly lower in the marathon athletes compared with the controls. Even though there were differences in BMI, the CRP levels were similar amongst the marathon runners. Thus, the authors suggested that lowered CRP levels can be attained by intense regular exercise, and that this suppression is independent of adiposity levels.

An interesting study by Mattusch et al. ${ }^{24}$ revealed changes in CRP concentration following 9 months of training. Fourteen males (25 - 40 years) preparing for the Cologne Marathon provided blood samples before and after 9 months of training for the event. The mean CRP concentration before the training began was $1.19 \pm 1.63 \mathrm{mg} / \mathrm{l}$ for athletes, and after 9 months was significantly $(p<0.05)$ reduced to $0.82 \pm 0.94 \mathrm{mg} / \mathrm{l}$. There were no significant changes reported for the control group. The authors suggested that an anti-inflammatory effect is induced by endurance exercise performed over 9 months. Similarly, Fallon et al. ${ }^{8}$ reported significantly $(p<$ 0.05 ) decreased levels of CRP following 9 months of soccer training in elite women, from a resting level of $2.68( \pm 1.70$ $\mathrm{mg} / \mathrm{l})$ to $1.62( \pm 1.32 \mathrm{mg} / \mathrm{l})$. It was suggested that systemic anti-inflammatory mechanisms, associated with regular intense exercise were behind this finding. The proposed antiinflammatory effect of chronic exercise suggests that physical activity may impart favourable health benefits on individuals by lowering CRP, a key role player in cardiovascular disease.

In conclusion, CRP is an acute-phase protein that is upregulated in response to injury, infection or antigen exposure. CRP usually peaks 24 hours after exercise, and is more pronounced following longer more strenuous activity. The elevations in CRP following exercise have largely been attributed to muscle damage, however, it seems plausible that muscle damage does not have to be elicited by exercise in order for CRP to be elevated. The alterations in CRP are closely related to exercise-induced inflammatory sequelae and are more pronounced following activity that is longer or more aerobically challenging than exercise that is not. The supposed lower resting levels and adaptations associated with chronic physical activity may be more prominent following aerobic exercise although studies are lacking in which the effects of resistance training are investigated. Numerous studies have shown that an inverse relationship exists between CRP and fitness levels, and that elevated CRP may be seen as a significant risk factor for CVD. In addition, resting CRP concentrations seem to be higher in sedentary versus active individuals. This supports the notion that exercise may 'downregulate' systemic inflammatory markers and thus prove beneficial in opposing chronic inflammatory disorders such as cardiovascular disease.

\section{REFERENCES}

1. Ablij $\mathrm{HC}$, Meinders $\mathrm{AE}$. C-reactive protein: history and revival. European Journal of Internal Medicine 2002; 13:412-22.

2. Benjamin E, Sunshine G, Leskowitz S. Immunology: A Short Course. New York: Wiley-Liss, 1996.

3. Berman S, Gerwuz H, Mold C. Binding of C-reactive protein to nucleated cells leads to complement activation without cytolysis. J Immunol 1986; 136:1354-9.

4. Cermak J, Key NS, Bach RR, Balla J, Jacob HS, Vercellotti GM. C-reactive protein induces human peripheral blood monocytes to synthesize tissue factor. Blood 1993; 82:513-20.

5. Church TS, Earnest CP, Wood KA, Kampert JB. Reduction of C-reactive protein levels through use of a multivitamin. Am J Med 2003; 115: 702-7.

6. Clyne B, Olshaker JS. The C-reactive protein. J Emerg Med 1999; 17:101925.

7. Du-Clos TW. Function of C-reactive protein. Ann Med 2000; 32: 274-8.

8. Fallon KE, Fallon SK, Boston T. The acute phase response and exercise: court and field sports. Br J Sports Med 2001; 35:170-3.

9. Gabay C, Kushner I. Acute phase proteins and other systemic responses to inflammation. N Engl J Med 1999; 340:448-54.

10. Ganapathi MK, Mackiewicz AJ, Samols D, et al. Induction of C-reactive protein by cytokines in human hepatoma cell lines is potentiated by caffeine. Biochem J 1990; 269: 41-6.

11.Gershov D, Kim S, Brot N, Elkon KB. C-reactive protein binds to apoptotic cells, protects the cells from assembly of the terminal complement components, and sustains an antiinflammatory innate immune response: Implications for systemic autoimmunity. J Exp Med 2000; 192:1353-64.

12. Gleeson M, Almey J, Brooks S, Cave R, Lewis A, Griffiths H. Heamatological and acute-phase responses associated with delayed onset muscle soreness. Eur J Appl Physiol 1995; 71:137-42.

13. Gould JM, Weiser JN. Expression of C-reactive protein in the human respiratory tract. Infect Immun 2001; 69:1747-54.

14.Ikuta $T$, Okubo $H$, Ishibashi $H$, Okumura $Y$, Hayashida K. Human lymphocytes synthesize C-reactive protein. Inflammation 1986; 10:223-32.

15. Jabs WJ, Logering BA, Gerke P, et al. The kidney as a second site of human C-reactive protein formation in vivo. Eur J Immunol 2003; 33:152-61.

16. Jeukendrup AE, Vet-Joop K, Sturk A, et al. Relationship between gastrointestinal complaints and endotoxaemia, cytokine release and the acute phase reaction during and after a long-distance triathlon in highly trained men. Clin Sci 2000; 98: 47-55

17.Ji LL. Antioxidants and oxidative stress in exercise. Experimental Biology and Medicine 1999; 222:283-92

18.King DE, Carek P, Mainous AG, Pearson WS. Inflammatory markers and exercise: Differences related to exercise type. Med Sci Sports Exerc 2003; 35:575-81.

19. Kushner I. C-reactive protein elevation can be caused by conditions other than inflammation and may reflect biologic ageing. Cleve Clin J Med 2001; 68:535-7

20.Kushner I, Rzewnicki D. The acute phase response: General aspects. Bail- 
lieres Clin Rheumatol 1994; 8: 513-30.

21. Lijnen P, Hespel P, Fagard R, et al. Indicators of cell breakdown in plasma of men during and after a marathon race. Int J Sports Med 1988; 9:108 13.

22. Maes M, Song C, Lin A, et al. The effects of psychological stress on humans: increased production of pro-inflammatory cytokines and a Th1-like response in stress-induced anxiety. Cytokine 1998; 10:313-8.

23. Malm C, Sjodin B, Sjoberg B, et al. Leukocytes, cytokines, growth factors and hormones in human skeletal muscle and blood after uphill or downhill running. J Physiol 2004; 556:983-1000.

24. Mattusch F, Dufaux B, Heine O, Mertens I, Rost R. Reduction of the plasma concentration of C-reactive protein following nine months of endurance training. Int J Sports Med 1999; 21:21-4.

25.McCarthy DA, Dale MM. The leucocytosis of exercise. A review and model. Sports Med 1988; 6:333-63.

26. Meyer TM, Gabriel H, Ratz M, Muller HJ, Kindermann W. Anaerobic exercise induces moderate acute phase response. Med Sci Sport Exer 2001; 33:549-55

27. Mold C, Gewurz H, Du-Clos TW. Regulation of complement activation by C-reactive protein. Immunopharmacology 1999; 42:23-30.

28. Morgan BP. Physiology and pathophysiology of complement: Progress and trends. Crit Rev Clin Lab Sci 1995; 32:265-98.

29. Nakagomi A, Freedman SB, Geczy CL. Interferon-gamma and lipopolysaccharide potentiate monocyte tissue factor induction by C-reactive protein Circulation 2000; 101:1785-91.

30. Nieman DC, Tan SA, Lee JW, Berk LS. Complement and immunoglobulin levels in athletes and sedentary controls. Int J Sports Med 1989;10:124-8.

31.Pasceri V, Chang J, Willerson JT, Yeh EHT. Modulation of C-reactive protein-mediated monocyte chemoattractant protein-1 induction in human endothelial cells by anti-atherosclerosis drugs. Circulation 2001; 103:2531-4.

32. Pasceri V, Willerson JT, Yeh EHT. Direct proinflammatory effect of C-reactive protein on human endothelial cells. Circulation 2000; 102: 2165-8.

33.Patel SS, Thiagarajan R, Willerson JT. Inhibition of alpha4-integrin and ICAM-1 markedly attenuate macrophage homing to artherosclerotic plaques in ApoE-deficient mice. Circulation 1998; 97:75-81.

34.Pepys MB. C-reactive protein: a critical update. J Clin Invest 2003; 111:1805-12.

35.Pepys MB, Baltz ML. Acute phase proteins with special reference to C-reactive protein and related proteins (pentaxins) and serum amyloid A-protein. Adv Immunol 1983; 34:141-211.

36. Pitsavos C, Chrysohoou C, Panagiotakos DB, et al. Association of leisuretime physical activity on inflammation markers (C-reactive protein, white cell blood count, serum amyloid $\mathrm{A}$, and fibrinogen) in healthy subjects. $\mathrm{Am}$ J Cardiol 2003; 91:368-70.

37.Pue CA, Mortensen RF, Marsh CB, Pope HA, Wewers MD. Acute levels of C-reactive protein enhance IL-1B and IL-1ra production by human blood monocytes but inhibit II-1B and IL-1ra production by alveolar macrophages. $J$ Immunol 1996; 156:1594-1600.

38. Ross R. Atherosclerosis - An inflammatory disease. N Engl J Med 1999; 340:115-26
39. Seifert PS, Kazatchkine MD. The complement system in atherosclerosis. Atherosclerosis 1988; 73:91-104.

40.Semple SJ, Smith LL, McKune AJ, Neveling N, Wadee AA. Alterations in acute-phase reactants (CRP, rheumatoid factor, complement, Factor $B$, and immune complexes) following an ultramarathon. South African Journal of Sports Medicine 2004; 16:17-21.

41. Shek PN, Shephard RJ. Physical exercise as a human model of limited inflammatory response. Can J Physiol Pharmacol 1998; 76:589-97.

42. Sherwood ER, Toliver-Kinsky T. Mechanisms of the inflammatory response. Best Practice and Research Clinical Anesthesiology 2004; 18:385-405.

43. Smith JA, Telford RD, Baker MS, Hapel AJ, Weidemann MJ. Cytokine immunoreactivity in plasma does not change after moderate endurance exercise. J Appl Physiol 1990; 73:1396-1401.

44. Sorichter S, Koller A, Haid C, et al. Light concentric exercise and heavy eccentric muscle loading: Effects on CK, MRI, and markers of inflammation. Int J Sports Med 1995; 16:288-92.

45. Suzuki K, Nakaji TMS, Yamada M, et al. Endurance exercise causes interaction among stress hormones, cytokines, neutrophil dynamics, and muscle damage. J Appl Physiol 1999; 87:1360-7.

46. Szeplaki G, Prohaszka Z, Duba J, et al. Association of high serum concentration of the third component of complement (C3) with pre-existing severe coronary artery disease and new vascular events in women. Atherosclerosis 2004; $177: 383-9$

47.Taylor C, Rogers G, Goodman C, et al. Hematologic, iron-related, and acute-phase protein responses to sustained strenuous exercise. J Appl Physiol 1987; 62:464-9.

48. Tillet WS, Francis T. Serological reactions in pneumonia with a non-protein somatic fraction of the Pneumococcus. J Exp Med 1930; 52:561-71.

49. Tomaszewski M, Charchar FJ, Crawford PM, et al. Strikingly low circulating CRP concentrations in ultramarathon runners independant of markers of obesity. How low can you go? Arterioscler Thromb Vasc Biol 2003; 23:1640-4.

50.Venugopal SK, Devaraj S, Yuhanna I, Shaul P, Jialal I. Demonstration that C-reactive protein decreases eNOS expression and bioactivity in human aortic endothelial cells. Circulation 2002; 106:1439-41.

51. Verma S, Wang $\mathrm{CH}$, Li SH, et al. C-reactive protein attentuates nitric oxide production and inhibits angiogenesis. Circulation 2002; 106:913-9.

52. Volanakis JE. Human C-reactive protein: expression, structure, and function. Mol Immunol 2001; 38:189-97.

53. Yasojima K, Schwab C, McGeer EG, McGeer PL. Generation of C-reactive protein and complement components in arthersclerotic plaques. $\mathrm{Am} \mathrm{J}$ Pathol 2001; 158:1039-51.

54.Zouki C, Beauchamp M, Baron C, Filep JG. Prevention of in vitro neutrophil adhesion to endothelial cells through shedding of L-selectin by C-reactive protein and peptides derived from C-reactive protein. J Clin Invest 1997; 100:522-9.

55.Zwaka TP, Hombach V, Torzewski J. C-reactive protein-mediated low density lipoprotein uptake by macrophages. Circulation 2001; 103:1194-7. 